\title{
Pulmonary arterial hypertension in limited cutaneous systemic sclerosis: a distinctive
} vasculopathy

\author{
M.J. Overbeek*, M.C. Vonk\#, A. Boonstra*, A.E. Voskuyl", A. Vonk-Noordegraaf*, \\ E.F. Smit*, B.A.C. Dijkmans ${ }^{\star}$, P.E. Postmus*, W.J. Mooi ${ }^{+}$, Y. Heijdra ${ }^{\S}$ and K. Grünberg ${ }^{+}$
}

ABSTRACT: Systemic sclerosis-associated pulmonary arterial hypertension (SScPAH) has a worse prognosis and response to pulmonary arterial hypertension (PAH) therapy than idiopathic PAH (IPAH). These differences have not yet been explained. Knowledge concerning histological pulmonary vasculopathy in SScPAH is limited in contrast to IPAH. Therefore, we explored patterns of vasculopathy in SScPAH compared with IPAH.

Parameters of vasculopathy were assessed from lung tissue of eight PAH patients with limited cutaneous systemic sclerosis and 11 IPAH patients. Lung tissue was obtained at autopsy $(n=15)$, explantation $(n=3)$ and biopsy $(n=1)$.

Pulmonary arterial/arteriolar intimal fibrosis was identified in all SScPAH patients and in three IPAH patients $(p=0.003)$. Fibrosis of pulmonary veins/venules was found in all SScPAH patients and in three IPAH patients $(p=0.003)$. In four SScPAH patients, fibrosis of veins/venules was focal and associated with capillary congestion as in pulmonary veno-occlusive disease (PVOD). Of the IPAH patients, 10 had unequivocal evidence of plexogenic arteriopathy compared with none of the SScPAH patients $(p=0.001)$.

SScPAH is characterised by small vessel intimal fibrosis, which is associated with a PVOD-like pattern in some cases. This might explain its different clinical behaviour from IPAH. Small vessel intimal fibrosis may provide clues to elucidation of differences in pathogenetic mechanisms between the groups.

KEYWORDS: Intimal fibrosis, plexiform lesion, pulmonary arterial hypertension, pulmonary veno-occlusive disease, systemic sclerosis

ystemic sclerosis (SSc) is an autoimmune disease characterised by the deposition of excessive amounts of extracellular matrix components, dysfunction of endothelium and an altered immune tolerance [1]. Patients with SSc are at risk of developing pulmonary arterial hypertension $(\mathrm{PAH})$, with estimated prevalence between $8-12 \%[2,3]$, which leads to right heart failure and death.

According to the third World Symposium on Pulmonary Arterial Hypertension in Venice, 2003, pulmonary hypertension $(\mathrm{PH})$ in SSc patients is classified in the $\mathrm{PAH}$ category, and subclassified in the category of PAH associated with collagen vascular diseases [4]. This categorisation is based on similarities of clinical presentation and therapy response. However, differences in clinical behaviour between $\mathrm{SScPAH}$ and other forms of PAH have been noted but have not yet been explained. First, in various reports $\mathrm{SScPAH}$ has a worse prognosis than idiopathic PAH (IPAH) [5, 6]. Secondly, patients with SScPAH present with lower values of transfer factor of the lung for carbon monoxide (TL,CO) than patients with IPAH [7, 8]. Thirdly, although responses to current $\mathrm{PAH}$ therapy have been reported to be effective in SScPAH $[9,10]$, some have reported less favourable responses as compared with IPAH [11-13]. These findings suggest that differences might, at least in part, be related to differences in associated vascular lesions. However, knowledge of SScPAH vascular morphology has remained limited, inherent to the rareness of the disease [14-18].

Herein, we will further explore the histopathological characteristics of pulmonary vasculopathy in a series of patients with clinically well-defined limited cutaneous SSc with documented PAH, and compare these to plexogenic arteriopathy in IPAH. IPAH is the most common representative

\section{AFFILIATIONS}

Depts of *Pulmonary Diseases,

"Rheumatology, and

+Pathology; VU University Medical

Center, Amsterdam,

Depts of ${ }^{\#}$ Rheumatology, and

${ }^{\S}$ Pulmonary Diseases, Radboud

University Nijmegen Medical Centre,

Nijmegen, The Netherlands.

\section{CORRESPONDENCE}

M.J. Overbeek

Dept of Pulmonary Diseases

VU University Medical Center

De Boelelaan 1117

P.0. Box 7057

$1007 \mathrm{MB}$

Amsterdam

The Netherlands

E-mail: mj.overbeek@VUmc.nl

Received:

July 142008

Accepted after revision:

March 052009

First published online:

March 262009 
of the 2003 WHO classification PAH group [17], and has a well described and homogeneous vascular morphology, with some similar features to those reported in SScPAH, such as concentric laminar intimal fibrosis and plexiform lesions [1921]. As clinical differences between the IPAH and SScPAH groups are well known, emerging differences in patterns of vascular lesions produced by these two entities may point to differences in pathogenesis and pathophysiology.

\section{MATERIALS AND METHODS \\ Case selection and review}

Cases were identified from the Depts of Pulmonary Diseases and Rheumatology of the VU University Medical Center (Amsterdam, the Netherlands) and the Depts of Rheumatology and Pulmonary Diseases of the Radboud University Nijmegen Medical Center (Nijmegen, the Netherlands). The study was approved by the Institutional Review Board on Research Involving Human Subjects of the VU University Medical Center (Amsterdam).

Patients were included if PAH was diagnosed by means of right heart catheterisation (RHC) (mean resting pulmonary arterial pressure $\left(\bar{P}_{\mathrm{pa}}\right) \geqslant 25 \mathrm{mmHg}$ and a pulmonary capillary wedge pressure $<15 \mathrm{mmHg}$ ). RHC data was unavailable for two IPAH patients as one patient refused a heart catheterisation and the other underwent RHC at another hospital but the results were not retrieved. At echocardiography, both patients had an elevated systolic pulmonary artery pressure (100 $\mathrm{mmHg}$ and $75 \mathrm{mmHg}$, respectively) without left atrial or left ventricular abnormalities. SSc cases were excluded if restrictive lung disease was present, as indicated by total lung capacity $<70 \%$ predicted, vital capacity $<70 \%$ and/or severe fibrosis on high-resolution computed tomography (HRCT) scan. SSc subset classification [22, 23], SSc disease duration and antibody profile were assessed.

For histological investigation, paraffin-embedded lung tissue samples from eight SScPAH patients and 11 IPAH patients were examined. Lung tissue was obtained at autopsy $(n=15)$, open lung biopsy $(n=1)$ or at lung transplantation $(n=3)$, which in all cases had been performed because of deterioration of PAH. Histological sections were stained with haematoxylin and eosin and Elastica von Gieson's. At least four blocks per patient were studied. Evaluation was performed using a standard semi-quantitative scoring sheet. The scoring of vascular morphology was performed by a histopathologist experienced in assessing pulmonary vascular histopathology (K. Grünberg). All scoring of cases was assessed within a 2week period, in a randomised order and the observer was blinded to the clinical diagnosis. A review of all the cases took place by both contributing pathologists, who were blinded to the clinical information and resulted in full agreement on specific vascular lesions (notably, pulmonary veno-occlusive disease (PVOD)-like pattern, concentric laminar intimal fibrosis, plexiform lesions).

\section{Scoring of histomorphology}

The lung tissue samples were evaluated for vasculopathy according to the consensus on assessing vasculopathy established at the third World Symposium on pulmonary hypertension [24] and according to the descriptions by WAGENVOORT and Moor [20] and Moor [25].
Media hyperplasia, intimal fibrosis and adventitial fibrosis were scored with reference to the type of vessel and microanatomical localisation, as were plexiform lesions, postthrombotic lesions, concentric laminar intimal fibrosis and vasculitis. Plexiform lesions were identified as arterial lesions consisting of a plexus of slit-like channels enclosed within or in continuity with a dilated segment of the affected artery [20]. Concentric laminar intimal fibrosis was identified on haematoxylin and eosin staining, based on an onionskin-type arrangement; care was taken not to mingle this configuration with the development of a new muscle coat within a layer of intimal fibrosis situated around the narrowed vessel lumen [20], or nonconcentric lamellar intimal fibrosis by loose connective tissue. In our analysis, PVOD and capillary haemangiomatosis was considered as one disease entity based on the clinical and morphological similarities [26]. Vasculitis/ vascular inflammation was defined as transmural infiltration of leukocytes (specified for type).

In addition to scoring vascular pathology, pulmonary interstitial fibrosis was scored and graded as none, mild (some interstitial fibrosis), moderate (architectural changes) and severe (honeycombing). Pneumonitis was described as none, sparse interstitial aggregates, mild diffuse interstitial infiltrates without fibrosis, moderate diffuse interstitial infiltrates, diffuse interstitial infiltrates with fibrosis, and infiltrates confined to fibrotic areas.

\section{Statistics}

A Mann-Whitney U-test was performed to compare differences in haemodynamic and respiratory parameters between the groups. Results are reported as median (range). Fisher's exact test was used for comparison of vascular histopathological parameters between groups.

A p value $<0.05$ was considered statistically significant. All analyses were performed with SPSS (version 12.0; SPSS, Chicago, IL, USA).

\section{RESULTS}

\section{Demographics}

Age was not significantly different between the groups (SScPAH 52 (32-60) versus IPAH 47 (23-59) yrs, p=0.90), nor was sex $(\mathrm{p}=0.63)$. Survival did not differ between the groups (SScPAH 0.75 (0.08-7) versus IPAH 2.75 (0.08-9) yrs, p=0.27). Characteristics of SSc patients are listed in table 1. All SSc patients fulfilled the preliminary American College of Rheumatology classification criteria for SSc and were classified as limited cutaneous SSc (LcSSc) [22, 23]. Mean disease duration from first non-Raynaud symptom of SSc to the time of PAH diagnosis was 10 yrs $(8(0.5-34)$ yrs). Of the autopsied patients, 14 had died of right ventricular failure and one SScPAH patient had died of hypovolumic shock due to iatrogenic bleeding (case 6). Three SSc patients demonstrated pericardial effusion at PAH diagnosis, compared with none of the IPAH patients. Macroscopic cardiac abnormalities at autopsy consisted mainly of right ventricular (RV) hypertrophy and dilatation. Of the three patients who underwent lung transplantation, one died post-operatively. In one SScPAH patient and one IPAH patient, macroscopic pulmonary emboli in the large vessels were found at post mortem examination. Data on reversibility [27] were acquired from five of the 
SScPAH and five of the IPAH patients; of these only three of the IPAH patients responded upon vasodilator therapy. At the time of death, most of the SScPAH and IPAH patients were being treated with prostacycline therapy (table 2).

\section{Clinical cardiopulmonary parameters}

Haemodynamic and pulmonary function parameters are listed in table 2. Values of cardiac index and pulmonary vascular resistance did not significantly differ, whereas $\bar{P}_{\text {pa }}$ values tended to be lower in the SSCPAH group compared with the IPAH group. No correlations with SSc characteristics, such as autoantibody profile and haemodynamic values, were found.

\section{Vasculopathy in SScPAH}

Parameters of vascular morphology for individual cases are outlined in table 3. Each single SSCPAH case demonstrated a spectrum of vasculopathic parameters. Several characteristic features were found in the majority of the $\mathrm{SSCPAH}$ patients.

Some degree of intimal fibrosis was found in the arterial tree at all levels in all patients. Notably, in all SScPAH cases, intimal fibrosis involving the small vessels at the level of alveolar parenchyma was observed (fig. 1). It should be noted that pulmonary arterioles and venules cannot be distinguished by the morphology of their walls: both consist of a layer of endothelium resting directly on a single elastic lamina. In many such small vessels, no connection to a larger, morphologically recognisable artery or vein was evident [20]. The pattern of intimal fibrosis of the small vessels dominated the overall picture of vascular morphology in four cases. In four SScPAH cases, the small vessel intimal fibrosis was associated with a pattern of PVOD (fig. 2). This pattern includes patchy intense capillary congestion in the alveolar parenchyma, and obliterative intimal fibrosis of small veins and venules. In three of these cases deposition of iron salts on elastin fibres, as well as haemosiderin-laden macrophages could be observed, also fitting in with capillary congestion and occult alveolar haemorrhage (fig. $2 b$ and c) [28]. In one case, a giant cell reaction to iron-encrusted elastin fibres was observed (fig. 2b).

\begin{tabular}{lc}
\hline TABLE 1 Characteristics of systemic sclerosis patients \\
Subjects & 8 \\
LcSSc & $8(100)$ \\
Disease duration ${ }^{\#}$ yr & $10 \pm 4$ \\
Antibody profile & \\
$\quad$ Anti-nuclear antibody & $8(100)$ \\
$\quad$ Anti-centromere & $6(75)$ \\
$\quad$ Anti-ribonucleoprotein & $1(12.5)$ \\
Anti-phospholipid auto-antibodies & 0 \\
Pericardial effusion & 3 \\
CT abnormalities associated with PVOD & 7 \\
Lymphadenopathy & 3 \\
Septal lines & 3 \\
Ground glass & 6 \\
\hline
\end{tabular}

Data are presented as $n, n$ (\%) or mean \pm SE. LCSSc: limited cutaneous systemic sclerosis; CT: computed tomography; PVOD: pulmonary veno-occlusive disease. ${ }^{\#}$ : since first non-Raynaud symptom, at time of diagnosis of pulmonary arterial hypertension.
In three out of these four SScPAH cases, this PVOD-like pattern was quite prominent, and hence designated the predominant pattern. It should be noted that such PVOD-like pattern is found in the setting of various other histopathological features, which include extensive intimal fibrosis, setting $\mathrm{SScPAH}$ apart from idiopathic PVOD. Intimal fibrosis of venules was observed in seven out of the eight SScPAH cases. These cases lacked the more diffuse congestion, interstitial fibrosis and fibrotic thickening of the walls of the large veins, indicative of congestive vasculopathy as seen, for example, in left heart failure or mitral valve insufficiency. In addition, there was no evidence of left-sided heart disease in the medical records including the autopsy reports.

Careful scrutiny of all available slides of the SScPAH patient group failed to reveal a single lesion as a convincing example of a plexiform lesion. The one lesion most closely resembling it is shown in figure 3a. Some dilatation lesions and slit-like channels are identified in this lesion, but it lacked the crowded endothelium with dark nuclei lining characterising plexiform lesions. It seems likely that the lesion represents a remnant of an organised thrombus.

Other vascular lesions in SScPAH cases included concentric laminar intimal fibrosis, observed in three of the SScPAH cases (fig. $4 \mathrm{a}$ and b). Concentric laminar intimal fibrosis was observed in the bronchiolar axial arteries and, in one case, also in the small parenchymal vessels. In one SScPAH case, this feature dominated other vasculopathic lesions. Loose concentric arterial intimal fibrosis was seen in seven of the $\mathrm{SScPAH}$ cases, in all of these cases it was seen in the small vessels; in four patients in veins and venules, in two patients in the bronchial and bronchiolar muscular arteries, and in one patient in both veins/venules and in bronchial/bronchiolar arteries. A pattern typical of hypoxic vasculopathy was not observed in any patient [20].

\section{Vasculopathy in SScPAH versus IPAH}

When comparing the previously mentioned features of the $\mathrm{SScPAH}$ cases with those characterising IPAH cases, some distinctions could be made. First, in general the intraindividual variety in SScPAH sections contrasted with the more homogenous aspect of vasculopathic features in IPAH sections; the vast majority of the latter demonstrated characteristics of plexogenic arteriopathy. The absence of plexiform lesions in $\mathrm{SScPAH}$ also distinguished this group from the IPAH group, in which 10 cases revealed plexiform lesions $(p=0.001)$ (fig. $3 b)$. None of the IPAH patients showed concentric laminar intimal fibrosis. Four IPAH cases did show diffuse hyperaemia. In two of these cases this could be related to vasodilatation, as they had been treated with prostacycline. Secondly, the involvement of intimal fibrosis in the small vessels in SScPAH appeared to be a diacritical feature of the two groups as this was only found in three of the $11 \mathrm{IPAH}$ patients $(\mathrm{p}=0.003)$. Again, in contrast to SSCPAH, a PVOD-like pattern could not be detected in any of the cases $(p=0.02)$. When analysis was confined to SSCPAH patients who did not demonstrate PVOD-like lesions, differences between these four patients and the IPAH group remained significant concerning the absence of plexiform lesions and the higher prevalence of small vessel intimal fibrosis $(p=0.004$ and $p=0.03$, respectively). Pulmonary venules and interlobular veins revealed 
TABLE 2 Haemodynamic, lung function and exercise data at pulmonary arterial hypertension (PAH) diagnosis

\begin{tabular}{|c|c|c|c|}
\hline & SScPAH & IPAH & $p$-value \\
\hline Subjects $n$ & 8 & 11 & \\
\hline Ppa,sys mmHg & $73(35-101) / 8$ & $100(62-128) / 11$ & 0.15 \\
\hline $\bar{P}_{\text {pa }} \mathrm{mmHg}$ & $45(25-71) / 8$ & $60(43-76) / 9$ & 0.08 \\
\hline PCWP $\mathrm{mmHg}$ & $7(4-12) / 8$ & $7(5-12) / 9$ & 0.57 \\
\hline FEV $1 \%$ pred & 88 (77-97) & $80(66-86)$ & 0.15 \\
\hline TLC \% pred & $88(81-115) / 6$ & $93(78-115) / 9$ & 0.78 \\
\hline vc \% pred & $97(84-115) / 7$ & $89(58-110) / 8$ & 0.34 \\
\hline$T L, C O \%$ pred & $40(26-58) / 7$ & $68(39-89) / 10$ & 0.01 \\
\hline$T L, C O / V_{A} \%$ & $39(26-69) / 7$ & $58.5(52-87) / 10$ & 0.01 \\
\hline $\mathrm{Pa}, \mathrm{O}_{2} \mathrm{mmHg}$ & $73(58-88)$ & $65.5(52-80)$ & 0.46 \\
\hline Prostacycline $n$ & 7 & 10 & \\
\hline ERA $n$ & 1 & 1 & \\
\hline PDE-5 inhibitor $n$ & 1 & & \\
\hline ABS $n$ & & 2 & \\
\hline
\end{tabular}

Data are presented as median (range)/n or median (range), unless otherwise stated. SScPAH: systemic sclerosis-associated PAH; IPAH: idiopathic PAH; $\bar{P}$ ra: mean right atrial pressure; Ppa,sys: systolic pulmonary artery pressure; $\bar{P}_{\text {pa: }}$ mean pulmonary artery pressure; PCWP: pulmonary capillary wedge pressure; PVR: pulmonary vascular resistance; $\mathrm{Cl}$ : cardiac index; FEV1; forced expiratory volume in $1 \mathrm{~s}$; \% pred: \% predicted; TLC: total long capacity; VC: vital capacity; $\mathrm{L}, \mathrm{CO}$ : transfer factor of the lung for carbon monoxide; VA: alveolar volume; $\mathrm{Pa}_{\mathrm{a}} \mathrm{O}_{2}$ : arterial oxygen tension; 6MWD: 6-min walk distance; ERA: endothelin receptor antagonist; PDE-5: phosphodiesterase 5; ABS: atrial balloon septostomy.

intimal fibrosis in all SScPAH cases, compared with three out of 11 IPAH patients, which was a significant difference $(\mathrm{p}=0.02)$. Finally, loose intimal fibrosis appeared to be a distinct characteristic, as it was observed in only three IPAH patients $(p=0.02)$

\section{Similarities in vasculopathy between SScPAH and IPAH}

All PAH cases demonstrated some degree of intimal fibrosis of the axial arteries at bronchiolar level. Medial hypertrophy of axial pulmonary arteries was observed in seven of the SScPAH cases and in 10 of the IPAH cases. Some degree of eccentric

TABLE 3 Parameters of pulmonary vascular morphology: systemic-sclerosis associated pulmonary arterial hypertension (PAH)

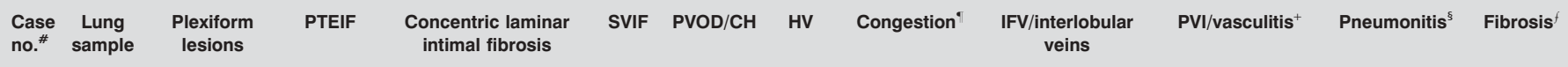

\begin{tabular}{|c|c|c|c|c|c|c|c|c|c|c|c|c|}
\hline 1 & LTX & $\mathrm{N}$ & $Y$ & $Y^{\# \#}$ & Y & $\mathrm{N}$ & $N$ & 1 & $N$ & 2 & 3 & 1 \\
\hline 2 & A & $\mathrm{N}$ & Y & $\mathrm{N}$ & Y & $Y^{\# \#}$ & $N$ & 1 & $Y$ & 1 & 1 & $\mathrm{~N}$ \\
\hline 3 & A & $\mathrm{N}$ & $\mathrm{N}$ & $\mathrm{N}$ & $Y^{\# \#}$ & $\mathrm{~N}$ & $\mathrm{~N}$ & $\mathrm{~N}$ & $Y$ & $1+3$ & 2 & $\mathrm{~N}$ \\
\hline 4 & A & $\mathrm{N}$ & Y & $\mathrm{N}$ & $Y$ & $\mathrm{Y}^{\# \#}$ & $\mathrm{~N}$ & 1 & $Y$ & $\mathrm{~N}$ & 1 & $\mathrm{~N}$ \\
\hline 5 & B & $\mathrm{N}$ & $Y$ & Y & Y & $\mathrm{N}$ & $N$ & $\mathrm{~N}$ & $Y$ & $\mathrm{~N}$ & 2 & $\mathrm{~N}$ \\
\hline 6 & A & $\mathrm{N}$ & Y & Y & Y & $Y^{\# \#}$ & $N$ & 2 & $Y$ & 1 & 2 & $\mathrm{~N}$ \\
\hline 7 & LTX & $\mathrm{N}$ & Y & $\mathrm{N}$ & $\mathrm{Y}^{\# \#}$ & $\mathrm{~N}$ & $\mathrm{~N}$ & $\mathrm{~N}$ & Y & $\mathrm{N}$ & 4 & $2+3$ \\
\hline 8 & A & $\mathrm{N}$ & Y & $\mathrm{N}$ & $\mathrm{Y}^{\# \#}$ & $\mathrm{Y}^{\# \#}$ & $N$ & 1 & $Y$ & 1 & $\mathrm{~N}$ & $\mathrm{~N}$ \\
\hline 9 & A & $\mathrm{N}$ & Y & $\mathrm{N}$ & $\mathrm{Y}^{\# \#}$ & $\mathrm{~N}$ & $N$ & $\mathrm{~N}$ & $N$ & 2 & 1 & $\mathrm{~N}$ \\
\hline 10 & A & $Y^{\# \#}$ & $Y$ & $\mathrm{~N}$ & $\mathrm{~N}$ & $\mathrm{~N}$ & $N$ & 3 & $N$ & 2 & 1 & $\mathrm{~N}$ \\
\hline 11 & LTX & $\mathrm{Y}^{\# \#}$ & Y & $\mathrm{N}$ & $\mathrm{N}$ & $\mathrm{N}$ & $\mathrm{N}$ & $\mathrm{N}$ & $\mathrm{N}$ & 2 & 1 & $\mathrm{~N}$ \\
\hline 12 & A & $\mathrm{Y}^{\# \#}$ & Y & $\mathrm{N}$ & $\mathrm{N}$ & $\mathrm{N}$ & $N$ & $1+3$ & $Y$ & 2 & $N$ & $\mathrm{~N}$ \\
\hline 13 & A & $Y^{\# \#}$ & $Y$ & $\mathrm{~N}$ & $\mathrm{~N}$ & $\mathrm{~N}$ & $N$ & $1+3$ & $N$ & $\mathrm{~N}$ & 1 & $\mathrm{~N}$ \\
\hline 14 & A & $\mathrm{Y}^{\# \#}$ & Y & $\mathrm{N}$ & Y & $\mathrm{N}$ & $N$ & $1+3$ & Y & 1 & $N$ & $\mathrm{~N}$ \\
\hline 15 & A & $\mathrm{Y}^{\# \#}$ & $\mathrm{Y}^{\# \#}$ & $\mathrm{~N}$ & $\mathrm{~N}$ & $\mathrm{~N}$ & $\mathrm{~N}$ & $\mathrm{~N}$ & $N$ & $\mathrm{~N}$ & 1 & $\mathrm{~N}$ \\
\hline 16 & A & $\mathrm{Y}^{\# \#}$ & Y & $\mathrm{N}$ & $\mathrm{N}$ & $\mathrm{N}$ & $N$ & $1+3$ & $N$ & $\mathrm{~N}$ & $\mathrm{~N}$ & $\mathrm{~N}$ \\
\hline 17 & A & $\mathrm{Y}^{\# \#}$ & Y & $\mathrm{N}$ & $\mathrm{N}$ & $\mathrm{N}$ & $N$ & $\mathrm{~N}$ & $Y$ & 1 & $\mathrm{~N}$ & $\mathrm{~N}$ \\
\hline 18 & A & $\mathrm{Y}^{\# \#}$ & Y & $\mathrm{N}$ & Y & $\mathrm{N}$ & $N$ & $\mathrm{~N}$ & $N$ & $\mathrm{~N}$ & 1 & $\mathrm{~N}$ \\
\hline 19 & A & $\mathrm{Y}^{\# \#}$ & $Y$ & $\mathrm{~N}$ & $\mathrm{~N}$ & $\mathrm{~N}$ & $\mathrm{~N}$ & $\mathrm{~N}$ & $N$ & $\mathrm{~N}$ & $\mathrm{~N}$ & $\mathrm{~N}$ \\
\hline
\end{tabular}

PTEIF: post-thrombotic eccentric intimal fibrosis; SVIF: small vessel intimal fibrosis; PVOD: pulmonary veno-occlusive disease; CH: capillary haemangiomatosis; HV: hypoxic vasculopathy; IFV: intimal fibrosis venules; PVI: pulmonary vascular inflammation. $\mathrm{N}$ : no; Y: yes; $\mathrm{A}$ : autopsy; $\mathrm{B}$ : biopsy. \#: cases 1 to 8 : systemic sclerosis-associated PAH; cases 9 to 19 : idiopathic PAH; $\because$ : $1=$ diffuse; $2=$ patchy; 3 =vasodilation related; LTX: lung explantation; ${ }^{+}: 1=$ lymphocytic; $2=$ perivascular infiltrate, insufficient for vasculitis; $3=$ granulocytic; ${ }^{\S}: 1=$ sparse interstitial aggregates; $2=$ diffuse interstitial infiltrate, mild, no fibrosis; $3=$ diffuse interstitial infiltrate, moderate, no fibrosis; $4=$ diffuse interstitial infiltrate and fibrosis; $5=$ infiltrate confined to fibrotic areas; ${ }^{f}: 1=$ mild, some interstitial fibrosis; 2 =moderate, architectural changes; $3=$ severe, honeycombing; $\# \#$ : predominant pattern. 

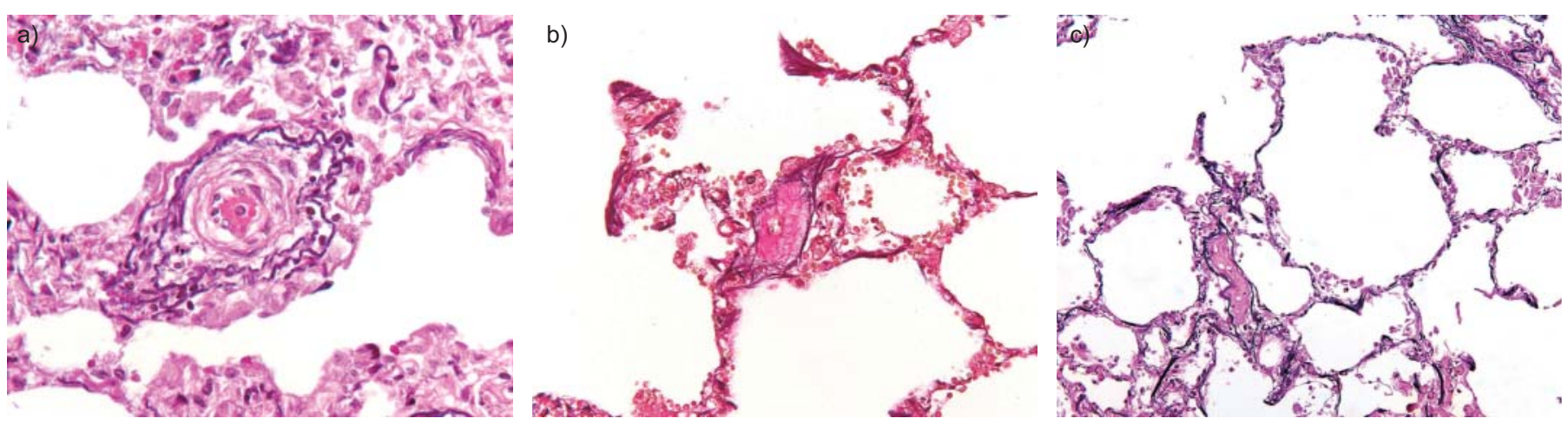

FIGURE 1. Intimal fibrosis of alveolar interstitial vessels. No distinction could be made between arteriole and venule on the basis of anatomic localisation (elastica von Gieson stain). a) Concentric intimal fibrosis (case 2, table 3) and b, c) complete obliteration (case 6, table 3). Original magnification: a and b) $\times 200, c) \times 100$

intimal fibrosis, indicative of post-thrombotic remodelling, was found in seven of the SSCPAH cases and in all IPAH cases. One $\mathrm{SScPAH}$ and one IPAH case demonstrated colander lesions; a recanalised thrombus reflecting post-thrombotic remodelling. In these two cases macroscopic thrombo-emboli were found at autopsy.

In four SScPAH and two IPAH cases we found vascular transmural infiltration of lymphocytes without evidence of destruction of the elastic laminae or fibrinoid changes of the vessel wall. In three of the SScPAH patients and one IPAH patient we observed this at the level of the small vessels. In one SScPAH case and one IPAH case the lymphocytic infiltrate appeared to be concentrated around the alveolar capillaries without evidence of haemorrhage as a sign of capillaritis. In two SScPAH cases, vasculitis was present at the venular level. In addition, in IPAH, mononuclear infiltrates were also detected in and around some plexiform lesions.

\section{Nonvascular morphological histological patterns in SScPAH and IPAH}

Areas of fibrosis were observed in two of the SScPAH cases, consisting of mild interstitial fibrosis in one patient and areas of fibrotic architectural changes and some honeycombing in the other patient. On most slides and in all cases, areas without fibrosis allowed for the evaluation of patterns of vasculopathy.
Pulmonary volumes, fibrosis on HRCT and peripheral oxygen saturation did not indicate pulmonary fibrosis as an underlying cause of PH. Seven of the SSCPAH cases and six of the $\mathrm{IPAH}$ cases showed some degree of pneumonitis. SScPAH cases demonstrated a pattern of diffuse interstitial infiltrates and of widening of the alveolar septa due to diffuse interstitial lymphocytic infiltrates, consistent with focal nonspecific interstitial pneumonia. In the two patients with areas of fibrosis, the inflammation was confined to fibrosis. Pneumonitis in IPAH cases was milder and consisted only of sparse interstitial infiltrates.

\section{DISCUSSION}

In this study we demonstrated that pulmonary vasculopathic morphology in SSCPAH displays heterogeneous features, but is dominated by the presence of intimal fibrosis of both arteries/ arterioles and veins/venules, which in some cases is associated with the presence of a PVOD-like pattern. This pattern of vasculopathy, together with the absence of plexiform lesions, sets apart the vasculopathy in SScPAH as an entity different from IPAH.

Previous studies have reported intimal fibrosis and medial hypertrophy in SSc with and without PAH [16, 18], and sometimes plexiform lesions [14], focusing on pulmonary precapillary lesions. There are few cases reported with PVOD associated with SSCPAH, in some cases, but not all, this has
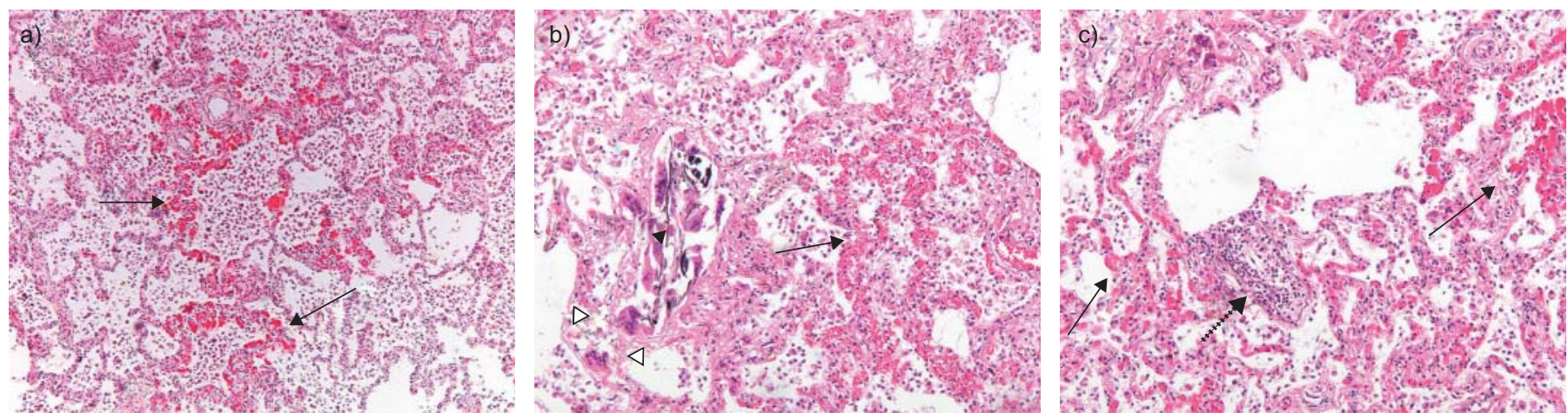

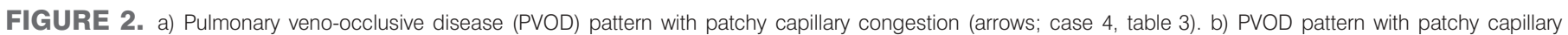

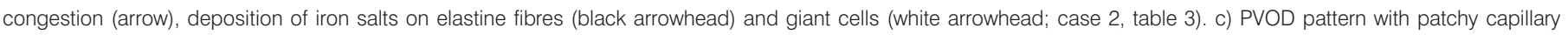

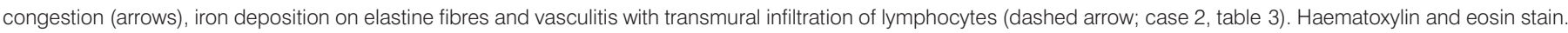
Original magnification: $\times 100$. 

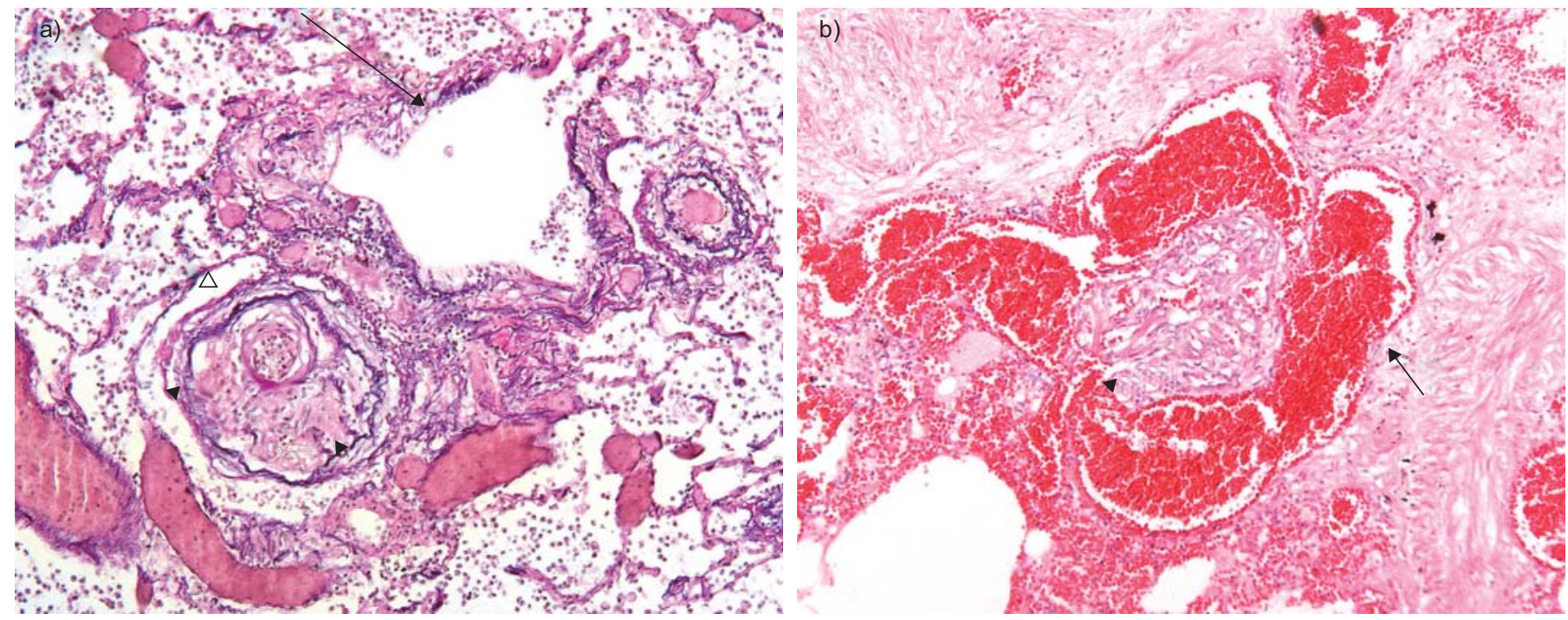

FIGURE 3. a) Single lesion in the systemic sclerosis-associated pulmonary arterial hypertension (PAH) group mostly resembling a plexiform lesion (case 3 , table 3) localisation adjacent to a bronchiolus (arrow); intimal fibrosis with recanalisation (black arrowheads); and dilated vessel segments (white arrowhead). b) Plexiform lesion in a patient with idiopathic PAH adjacent to a feeding artery, with intimal fibrosis and slit-like channels lined by cuboidal endothelial cells (arrowhead) and dilated vessel segments (arrow; case 19, table 3). a) elastica von Gieson stain, b) haematoxylin and eosin stain. Original magnification: $\times 100$

been proven by biopsy [29-31]. A recent study by DORFMÜLLER et al. [15] involving eight connective tissue disease (CTD) cases with clinically well defined PAH, including four LcSSc patients, demonstrated a PVOD-like pattern in the CTD population, together with post-capillary fibrous involvement and signs of alveolar haemorrhagic oedema. Our study, describing, to date, the largest series of patients of clinically well defined SSc patients with thoroughly documented $\mathrm{PAH}$, confirms these findings and extends upon them by demonstrating small vessel intimal fibrosis, coinciding with a pattern of PVOD in some cases, and indicating arteriolar as well as venular intimal involvement.

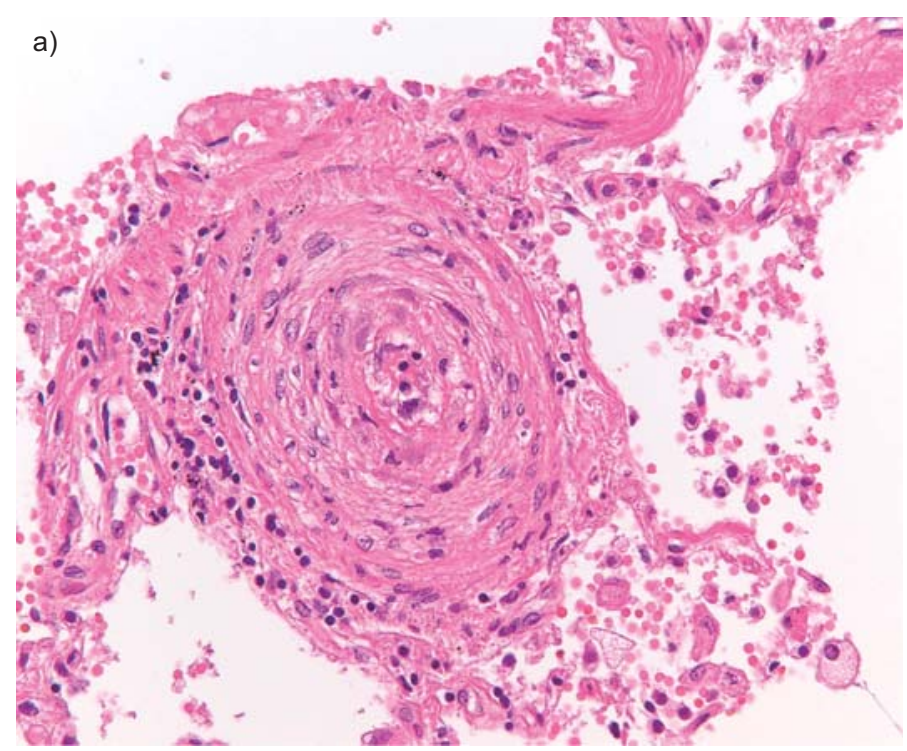

Interestingly, in SSc without $\mathrm{PAH}$, the arterial and venous vessels of small calibre, as well as the microcirculation in SScaffected hands, demonstrated microvascular involvement [32]. A report of 17 patients with the CREST syndrome (a formerly used term recognised as a variant of SSc characterised by calcinosis, Raynaud's phenomenon, oesophageal dismotility, sclerodactyly and telangiectasis) which, according to present standards defined PAH inadequately, described fibrotic alterations in pulmonary venules in cases both with and without PAH [18]. We observed a similar spectrum of pulmonary vasculopathy in two SSc patients without pulmonary hypertension (data not shown).

FIGURE 4. a, b) Pulmonary artery with concentric laminar intimal fibrosis and some perivascular mononuclear cell infiltration (case 6, table 3). a) Haematoxylin and eosin stain, b) elastica von Gieson stain. Original magnification: $\times 200$.

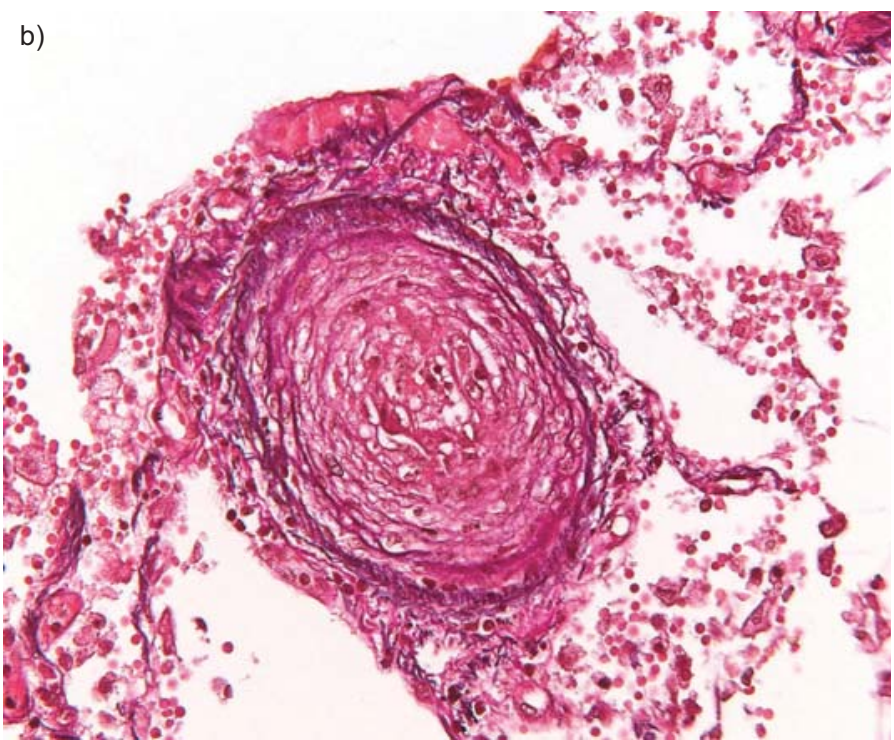


Convincing examples of plexiform lesions were not detected in the SScPAH group. Some authors have reported the absence of plexiform lesions [15], whereas others have reported the occurrence of such lesions [14]. As such, some controversy concerning this topic exists. It is unlikely that in our study interpretations of morphology of plexiform lesions versus thrombo-embolic lesions explain this discrepancy, as both types of lesions were rare in our SScPAH group. Rather, in our opinion, the absence of plexiform lesions is another diacritical feature of SScPAH versus IPAH.

In our study, concentric laminar fibrosis was strictly defined according to previously described criteria [20], whereas an apparently broader definition allowed inclusion of concentric intimal fibrosis consisting of loose fibrous tissue [15]. Concentric laminar intimal fibrosis was observed in three out of the eight SScPAH cases, whereas in the study of DORFMÜLLER et al. [15] it was observed in three out of four SScPAH cases. Indeed, we observed loose intimal fibrosis in the majority of the SScPAH cases. We did not find concentric laminar fibrosis in our IPAH cases, whereas loose intimal fibrosis was found in two cases. The significance of distinguishing concentric laminar intimal fibrosis from concentric intimal fibrosis may be a matter of debate: whether this lesion represents a distinct entity or whether it represents a stage, or the end result, of intimal fibrosis remains to be determined.

The present data were largely based on autopsy material. The inherent limitations of such a study design are shared by previous studies on this topic. As nearly all the patients died of PAH-related causes, the pathology in our study represents endstage disease. Consequently, its features may differ from earlier phases of the same disease. However, histopathology evolves over time, and this study demonstrates that during similar disease duration of $\mathrm{PAH}$, the groups end up with different histopathological patterns. This suggests that different pathogenetic pathways are involved. The SScPAH population consisted of patients with LcSSc [23], with none or mild interstitial disease. As data on pulmonary vascular changes in SSc with fibrosis are scare, it is currently not clear whether our results can be extrapolated to the SSc patients, either LcSSc or diffuse cuatneous SSc, with major intersitial lung disease. In contrast to previous reports, in our study survival was not significantly different, whereas SSCPAH patients are known to have a worse prognosis than IPAH patients, even despite treatment $[5,6]$. It should be noted that the study was not designed to address this issue, and the sample size is too small to draw firm conclusions on this matter. However, one could postulate that selection may have been biased by recruitment from tertiary referral centres with high awareness for PAH in SSc patients.

How can these findings be interpreted? Explanations of the difference in arteriolar and venular intimal fibrosis between the groups are speculative, but might be related to the systemic nature of SSc disease. For example, mediators of endothelial dysfunction that influence vascular remodeling and fibrogenesis, such as endothelin-1, play an important role in SSc pathogenetics [33, 34]. The perivascular and transmural infiltration of lymphocytes, both in SScPAH as well as in IPAH, and the surrounding of plexiform lesions by lymphocytes is in line with previous observations by others, supporting the notion that inflammatory mechanisms may be relevant in the pathology of PAH [35-37]. Various studies describe specific inflammatory mechanisms concerning SSCPAH, such as a different T-cell/endothelium interplay from SSc patients with and without PAH, and the occurrence of anti-endothelial cell and anti-fibroblast antibodies [38-42]. In our study, in both $\mathrm{SScPAH}$ and IPAH, we observed perivascular and transmural infiltration of lymphocytes, as well as surrounding of plexiform lesions by such infiltrates. Taken together, selective intimal fibrosis might be a reflection of the inflammatory nature of SSc.

In a recent study by MONTANI et al. [43], precipitous pulmonary oedema with initiation of PAH therapy occurred in $44 \%$ of patients with PVOD. Therefore, SScPAH patients who demonstrate PVOD-like pattern might be susceptible of developing this complication. Interestingly, cases have been reported of SScPAH patients developing pulmonary oedema upon epoprostenol administration [44, 45]. However, in our population no pulmonary oedema occurred at initiation of prostacyclines or at testing of vasodilation upon nitric oxide. Whether the presence of PVOD-like pattern raises the susceptibility of these patients to pulmonary oedema needs to be confirmed in larger series.

Small vessel intimal fibrosis and the PVOD pattern occurring at this background may account for the different response to therapy in SScPAH as compared with IPAH [46]. Moreover, it could be speculated that this phenomenon explains the lower $T \mathrm{~L}, \mathrm{CO}$ values in SSCPAH than in IPAH, as post-capillary abnormalities may lead to disturbed gas diffusion at the capillary level [47]. Indeed, low TL,CO is also a feature of clinical PVOD [46].

It is a matter of speculation to explain the absence of plexiform lesions in SScPAH. Altered angiogenesis, as seen in SSc [48], might play a role as, for example, it has been demonstrated in SSc upregulation of vascular endothelial growth factor (VEGF) and its receptor (VEGFR) [49]. Interestingly, in plexiform lesions, upregulation of the VEGF/VEGFR axis has been shown [50-52] and as such they are thought to arise from uncontrolled angiogenesis. In this context, it is intriguing that plexiform lesions were not observed in SScPAH in the reported cases, challenging in depth study.

Taken together, this study demonstrates that pulmonary vasculopathic features in SSCPAH differ from those seen in IPAH with respect to the occurrence of small vessel intimal fibrosis, a pattern of PVOD and absence of plexiform lesions. These findings may account for differences in terms of $T \mathrm{~L}, \mathrm{CO}$ values and response to $\mathrm{PAH}$ therapy between the groups. Moreover, this study supports the notion, as evoked by differences in clinical behaviour, that different pathogenetic mechanisms may underlie the development of PAH. This study underpins the importance of further research into targets for tailored treatment for SSc-associated PAH.

\section{STATEMENT OF INTEREST}

Statements of interest for A. Boonstra and A. Vonk-Noordegraaf can be found at www.erj.ersjournals.com/misc/statements.dtl

\section{REFERENCES}

1 Jimenez SA, Derk CT. Following the molecular pathways toward an understanding of the pathogenesis of systemic sclerosis. Ann Intern Med 2004; 140: 37-50. 
2 Hachulla E, Gressin V, Guillevin L, et al. Early detection of pulmonary arterial hypertension in systemic sclerosis: a French nationwide prospective multicenter study. Arthritis Rheum 2005; 52: 3792-3800.

3 Mukerjee D, St George D, Coleiro B, et al. Prevalence and outcome in systemic sclerosis associated pulmonary arterial hypertension: application of a registry approach. Ann Rheum Dis 2003; 62: 1088-1093.

4 Simonneau G, Galie N, Rubin LJ, et al. Clinical classification of pulmonary hypertension. J Am Coll Cardiol 2004; 43: Suppl. 12, 5S-12S.

5 Fisher MR, Mathai SC, Champion HC, et al. Clinical differences between idiopathic and scleroderma-related pulmonary hypertension. Arthritis Rheum 2006; 54: 3043-3050.

6 Kawut SM, Taichman DB, Archer-Chicko CL, et al. Hemodynamics and survival in patients with pulmonary arterial hypertension related to systemic sclerosis. Chest 2003; 123: 344-350.

7 Steen V, Medsger TA Jr. Predictors of isolated pulmonary hypertension in patients with systemic sclerosis and limited cutaneous involvement. Arthritis Rheum 2003; 48: 516-522.

8 Sun XG, Hansen JE, Oudiz RJ, et al. Pulmonary function in primary pulmonary hypertension. J Am Coll Cardiol 2003; 41: 1028-1035.

9 Badesch DB, Tapson VF, McGoon MD, et al. Continuous intravenous epoprostenol for pulmonary hypertension due to the scleroderma spectrum of disease. A randomized, controlled trial. Ann Intern Med 2000; 132: 425-434.

10 Badesch DB, Hill NS, Burgess G, et al. Sildenafil for pulmonary arterial hypertension associated with connective tissue disease. J Rheumatol 2007; 34: 2417-2422.

11 Girgis RE, Mathai SC, Krishnan JA, et al. Long-term outcome of bosentan treatment in idiopathic pulmonary arterial hypertension and pulmonary arterial hypertension associated with the scleroderma spectrum of diseases. J Heart Lung Transplant 2005; 24: 1626-1631.

12 Mathai SC, Girgis RE, Fisher MR, et al. Addition of sildenafil to bosentan monotherapy in pulmonary arterial hypertension. Eur Respir J 2007; 29: 469-475.

13 Rubin LJ, Badesch DB, Barst RJ, et al. Bosentan therapy for pulmonary arterial hypertension 1. N Engl J Med 2002; 346: 896-903.

14 Cool CD, Kennedy D, Voelkel NF, et al. Pathogenesis and evolution of plexiform lesions in pulmonary hypertension associated with scleroderma and human immunodeficiency virus infection. Hum Pathol 1997; 28: 434-442.

15 Dorfmuller P, Humbert M, Perros F, et al. Fibrous remodeling of the pulmonary venous system in pulmonary arterial hypertension associated with connective tissue diseases. Hum Pathol 2007; 38: 893-902.

16 al Sabbagh MR, Steen VD, Zee BC, et al. Pulmonary arterial histology and morphometry in systemic sclerosis: a case-control autopsy study. J Rheumatol 1989; 16: 1038-1042.

17 Peacock AJ, Murphy NF, McMurray JJ, et al. An epidemiological study of pulmonary arterial hypertension. Eur Respir J 2007; 30: 104-109.

18 Yousem SA. The pulmonary pathologic manifestations of the CREST syndrome. Hum Pathol 1990; 21: 467-474.

19 Pietra GG, Edwards WD, Kay JM, et al. Histopathology of primary pulmonary hypertension. A qualitative and quantitative study of pulmonary blood vessels from 58 patients in the National Heart, Lung, and Blood Institute, Primary Pulmonary Hypertension Registry. Circulation 1989; 80: 1198-1206.

20 Wagenvoort CA, Mooi WJ. Biopsy Pathology of the Pulmonary Vasculature. Biopsy Pathology Series. 1st Edn. London, Chapman and Hall Medical, 1989.

21 Wagenvoort CT, Wagenvoort N. Primary pulmonary hypertension; a pathologic study of the lung vessels in 156 clinically diagnosed cases. Circulation 1970; 42: 1163-1184.

22 Preliminary criteria for the classification of systemic sclerosis (scleroderma). Subcommittee for scleroderma criteria of the
American Rheumatism Association Diagnostic and Therapeutic Criteria Committee. Arthritis Rheum 1980; 23: 581-590.

23 LeRoy EC, Black C, Fleischmajer R, et al. Scleroderma (systemic sclerosis): classification, subsets and pathogenesis. I Rheumatol 1988; 15: 202-205.

24 Pietra GG, Capron F, Stewart S, et al. Pathologic assessment of vasculopathies in pulmonary hypertension. J Am Coll Cardiol 2004; 43: Suppl. 12, 25S-32S.

25 Mooi WJ, Grünberg K. Histopathology of pulmonary hypertensive diseases. Curr Diagn Pathol 2006; 12: 429-440.

26 Lantuejoul S, Sheppard MN, Corrin B, et al. Pulmonary venoocclusive disease and pulmonary capillary hemangiomatosis: a clinicopathologic study of 35 cases. Am J Surg Pathol 2006; 30: 850-857.

27 Badesch DB, Abman SH, Simonneau G, et al. Medical therapy for pulmonary arterial hypertension: updated ACCP evidence-based clinical practice guidelines. Chest 2007; 131: 1917-1928.

28 Rabiller A, Jais X, Hamid A, et al. Occult alveolar haemorrhage in pulmonary veno-occlusive disease. Eur Respir J 2006; 27: 108-113.

29 Johnson SR, Patsios D, Hwang DM, et al. Pulmonary venoocclusive disease and scleroderma associated pulmonary hypertension. J Rheumatol 2006; 33: 2347-2350.

30 Morassut PA, Walley VM, Smith CD. Pulmonary veno-occlusive disease and the CREST variant of scleroderma. Can J Cardiol 1992; 8: 1055-1058.

31 Saito A, Takizawa $\mathrm{H}$, Ito $\mathrm{K}$, et al. A case of pulmonary venoocclusive disease associated with systemic sclerosis. Respirology 2003; 8: 383-385.

32 Allanore $\mathrm{Y}$, Seror R, Chevrot A, et al. Hand vascular involvement assessed by magnetic resonance angiography in systemic sclerosis. Arthritis Rheum 2007; 56: 2747-2754.

33 Abraham D, Distler O. How does endothelial cell injury start? The role of endothelin in systemic sclerosis. Arthritis Res Ther 2007; 9 ; Suppl. 2, S2.

34 Ahmed SS, Tan FK, Arnett FC, et al. Induction of apoptosis and fibrillin 1 expression in human dermal endothelial cells by scleroderma sera containing anti-endothelial cell antibodies. Arthritis Rheum 2006; 54: 2250-2262.

35 Dorfmuller P, Perros F, Balabanian K, et al. Inflammation in pulmonary arterial hypertension. Eur Respir J 2003; 22: 358-363.

36 Nicolls MR, Taraseviciene-Stewart L, Rai PR, et al. Autoimmunity and pulmonary hypertension: a perspective. Eur Respir J 2005; 26: 1110-1118.

37 Perros F, Dorfmuller P, Souza R, et al. Dendritic cell recruitment in lesions of human and experimental pulmonary hypertension. Eur Respir J 2007; 29: 462-468.

38 Baroni SS, Santillo M, Bevilacqua F, et al. Stimulatory autoantibodies to the PDGF receptor in systemic sclerosis. $N$ Engl J Med 2006; 354: 2667-2676.

39 Carulli MT, Handler C, Coghlan JG, et al. Can CCL2 serum levels be used in risk stratification or to monitor treatment response in systemic sclerosis? Ann Rheum Dis 2008; 67: 105-109.

40 Chizzolini C, Raschi E, Rezzonico R, et al. Autoantibodies to fibroblasts induce a proadhesive and proinflammatory fibroblast phenotype in patients with systemic sclerosis. Arthritis Rheum 2002; 46: 1602-1613.

41 Sanchez O, Marcos E, Perros F, et al. Role of endothelium-derived CC chemokine ligand 2 in idiopathic pulmonary arterial hypertension. Am J Respir Crit Care Med 2007; 176: 1041-1047.

42 Tamby MC, Humbert M, Guilpain P, et al. Antibodies to fibroblasts in idiopathic and scleroderma-associated pulmonary hypertension. Eur Respir J 2006; 28: 799-807.

43 Montani D, Achouh L, Dorfmuller P, et al. Pulmonary venoocclusive disease: clinical, functional, radiologic, and hemodynamic characteristics and outcome of 24 cases confirmed by histology. Medicine (Baltimore) 2008; 87: 220-233. 
44 Humbert M, Sanchez O, Fartoukh M, et al. Short-term and longterm epoprostenol (prostacyclin) therapy in pulmonary hypertension secondary to connective tissue diseases: results of a pilot study. Eur Respir J 1999; 13: 1351-1356.

45 Preston IR, Klinger JR, Houtchens J, et al. Pulmonary edema caused by inhaled nitric oxide therapy in two patients with pulmonary hypertension associated with the CREST syndrome. Chest 2002; 121: 656-659.

46 Mandel J, Mark EJ, Hales CA. Pulmonary veno-occlusive disease. Am J Respir Crit Care Med 2000; 162: 1964-1973.

47 Hughes JM. The single breath transfer factor $(T L, C O)$ and the transfer coefficient (KCO): a window onto the pulmonary microcirculation. Clin Physiol Funct Imaging 2003; 23: 63-71.

48 Koch AE, Distler O. Vasculopathy and disordered angiogenesis in selected rheumatic diseases: rheumatoid arthritis and systemic sclerosis. Arthritis Res Ther 2007; 9: Suppl. 2, S3.
49 Distler O, Distler JH, Scheid A, et al. Uncontrolled expression of vascular endothelial growth factor and its receptors leads to insufficient skin angiogenesis in patients with systemic sclerosis. Circ Res 2004; 95: 109-116.

50 Cool CD, Stewart JS, Werahera P, et al. Three-dimensional reconstruction of pulmonary arteries in plexiform pulmonary hypertension using cell-specific markers. Evidence for a dynamic and heterogeneous process of pulmonary endothelial cell growth. Am J Pathol 1999; 155: 411-419.

51 Lee SD, Shroyer KR, Markham NE, et al. Monoclonal endothelial cell proliferation is present in primary but not secondary pulmonary hypertension. J Clin Invest 1998; 101: 927-934.

52 Tuder RM, Chacon M, Alger L, et al. Expression of angiogenesisrelated molecules in plexiform lesions in severe pulmonary hypertension: evidence for a process of disordered angiogenesis. J Pathol 2001; 195: 367-374 